\title{
CARACTERIZACIÓN DE SENSORES PARA APLICACIONES EN BIOMECÁNICA
}

Lautaro VERA; Oscar G. LOMBARDERO (*)

\section{RESUMEN}

Con el auge de los Sistemas Microelectromecánicos (MEMS), la relación capacidad/tamaño de los sensores tuvo un crecimiento exponencial, tomando un papel principal en la investigación de medicina y rehabilitación, como por ejemplo en el registro del movimiento asociado al cuerpo humano.

En el presente trabajo se presenta la caracterización del comportamiento de un acelerómetro y un giróscopo, ambos de 3 ejes, integrados en la IMU (Inertial Measurement Unit) MPU-6050 de InvenSense ${ }^{\mathrm{TM}}$, que en hipótesis son capaces de establecer la orientación de una masa respecto al plano de referencia del dispositivo.

Para comprobar esta hipótesis, se somete al dispositivo a exhaustivos ensayos tanto estáticos como dinámicos, para poder determinar linealidad, precisión, exactitud y los errores inherentes que poseen.

\section{INTRODUCCIÓN}

Los estudios de biomecánica suponen un complemento fundamental para el profesional de la salud a la hora de evaluar cuantitativamente la capacidad funcional de los miembros involucrados en el movimiento del cuerpo humano.

Para poder precisar un diagnóstico correcto y elaborar un tratamiento óptimo, se debe recurrir a un enfoque interdisciplinario, que involucre tanto las ciencias médicas como los desarrollos particulares de ingeniería.

El objetivo de este trabajo fue establecer si es posible utilizar sensores inerciales, más precisamente un acelerómetro y un giróscopo, como indicadores de posición en forma estática y dinámica. Para esto se buscó caracterizarlos en cuanto a su comportamiento, y de esa forma estimar el alcance de los resultados.

A priori se conoce por el principio de funcionamiento de dichos sensores, que su capacidad está limitada a describir la posición de un cuerpo respecto a un estado inicial de reposo. Es decir, se pueden precisar inclinaciones, rotaciones, pero no se puede precisar si el cuerpo se desplazó de un punto a otro. Este tipo de sensores están diseñados para mediciones biomecánicas, donde se busca conocer rotaciones e inclinaciones producidas por un movimiento aproximadamente uniforme, y resulta indistinto en

(*) GRIER Grupo de Ingeniería en Rehabilitación. FACENA UNNE. 
qué punto de la trayectoria se encuentra el cuerpo bajo estudio.

\section{MATERIALES Y MÉTODOS}

\section{Dispositivos Utilizados}

Para mayor versatilidad, los fabricantes colocan los sensores dentro de placas de adaptación (breakout board), que contienen varios circuitos de apoyo. La placa de adaptación en el caso del MPU-6050 es la GY521 , y es la que se utilizó en el proyecto.

El módulo GY-521 ofrece salida digital mediante el protocolo $\mathrm{I}^{2} \mathrm{C}$, conversores analógico/digital de 16 bits de resolución por cada canal tanto del acelerómetro como del giróscopo (capta los ejes $\mathrm{x}, \mathrm{y}, \mathrm{z}$ al mismo tiempo), y más de 100 registros. Parte de estos registros almacenan las mediciones digitalizadas por los conversores en un instante determinado, y se conservan para una comunicación $\mathrm{I}^{2} \mathrm{C}$. Otra parte de los registros establecen la configuración de los sensores, como sensibilidad, frecuencia de corte del filtro pasa bajos interno, etc. Ambos, acelerómetro y giróscopo tienen 4 sensibilidades seleccionables: $\pm 2 \mathrm{~g}, \pm 4 \mathrm{~g}, \pm 8 \mathrm{~g}, \pm 16 \mathrm{~g}$ $\mathrm{y} \pm 250 \mathrm{dps}, \pm 500 \mathrm{dps}, \pm 1000 \mathrm{dps}, \pm 2000 \mathrm{dps}$. Estas son las características principales, en la hoja de datos se exponen con mayor detalle todas sus características.

El principio de funcionamiento del acelerómetro es capacitivo y mide la aceleración asociada con el fenómeno de peso que experimenta una masa de prueba dentro del marco de referencia del dispositivo. El principio de funcionamiento del giróscopo es de estructura vibrante y mide la velocidad angular basándose en la fuerza de Coriolis que afecta a dicha estructura. Por si solos, no funcionan como indicadores de posición, por lo tanto es necesario establecer una interfaz con un microcontrolador para el procesamiento de los datos, y transformarla en información útil.

Como microcontrolador se utilizó un ATmega328P (Arduino Nano) y para conservar los datos se utilizó una memoria EEPROM AT24C04C, ambos de Atmel ${ }^{\mathrm{TM}}$.

Fig. 1. Dispositivo Final, en la imagen se visualiza los componentes involucrados y el panel de control

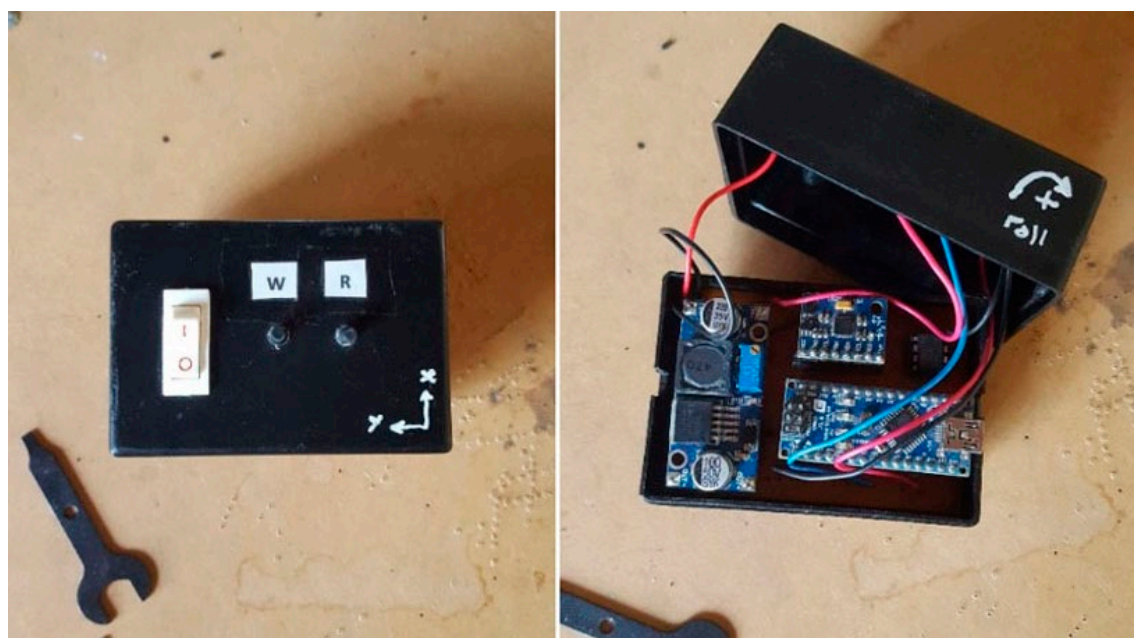


Se eligió esta memoria por tener comunicación $\mathrm{I}^{2} \mathrm{C}$, minimizando la cantidad de conexionado y potenciando la portabilidad, ya que los ensayos requerían un dispositivo final inalámbrico.

Cuatro baterías recargables $\mathrm{Ni}-\mathrm{MH}$ de $1.2 \mathrm{~V}$ y $1100 \mathrm{mAh}$ en serie son las encargadas de energizar los circuitos. El Arduino Nano utiliza un regulador de tensión similar al 7805, por lo tanto necesita un voltaje de "dropout" mínimo de $2 \mathrm{~V}$ aproximadamente para regular correctamente 5V. Para alcanzar los $7 \mathrm{~V}$ se utiliza la fuente step-up DCDC modelo CN6009 de alta eficiencia.
El giróscopo, en cambio, cuenta con alta inmunidad al ruido, posee un mayor ancho de banda $(8 \mathrm{kHz})$ y sigue correctamente eventos de mayor frecuencia. No tiene tanta exactitud como el acelerómetro, y es propenso al error de deriva (drift).

Los valores de ancho de banda son teóricos y son dados por el fabricante en la hoja de datos. Los estudios de biomecánica involucran frecuencias relativamente bajas y a priori los eventos lentos pueden ser determinados con exactitud por el acelerómetro, y los eventos rápidos seguidos correctamente por el giróscopo.

\section{Errores inherentes}

En este apartado se describen los errores propios tanto del acelerómetro como del giróscopo dentro del módulo MPU-6050. Esta clase de imprecisiones dependen exclusivamente de la construcción del instrumento y del principio de funcionamiento que lo gobierna.

$\mathrm{E}$ l acelerómetro es altamente sensible al ruido eléctrico, y en menor medida al ruido mecánico. No está pensado para detectar vibraciones, pero puede detectar vibraciones no deseadas no tan pequeñas, $y$ formaran parte del ruido intrínseco del mismo. Además, tiene un ancho de banda reducido $(1 \mathrm{kHz})$, funcionando mejor a frecuencias bajas. No tiene la capacidad de seguir eventos de mayor frecuencia (para ello se prefiere el giróscopo). A favor del acelerómetro, es muy preciso y cuenta con elevada exactitud.

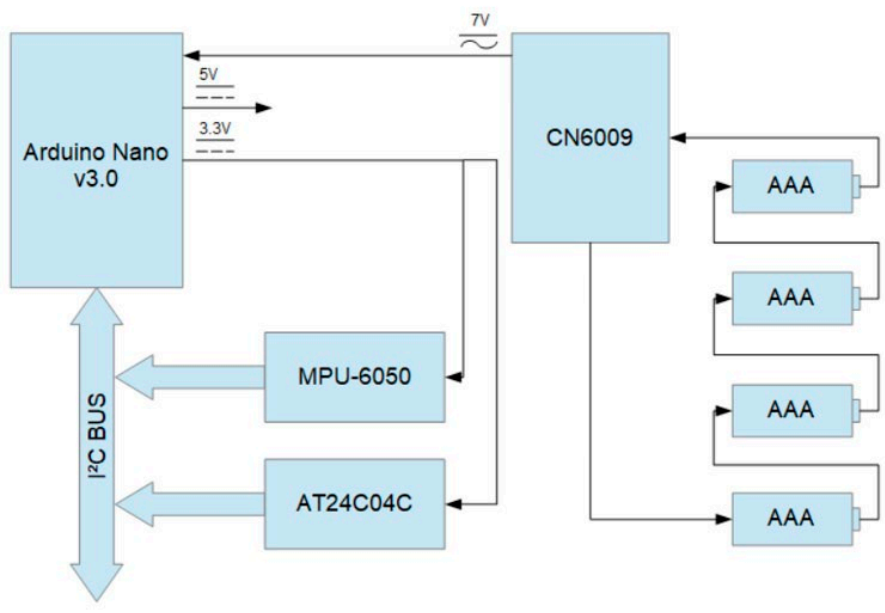

Fig. 2. Diagrama en Bloques del conexionado de los componentes

\section{Circuitos Implementados}

En la Figura 2 se esque-matiza la conexión de los dispositivos presentados anteriormente. El circuito impreso correspondiente se desarrolló en una placa de $5 \times 7 \mathrm{~cm}$, las cuales son las mismas dimensiones de la caja contenedora. En la parte superior de la misma se presenta un panel con un interruptor de encendido/apagado y dos pul- 


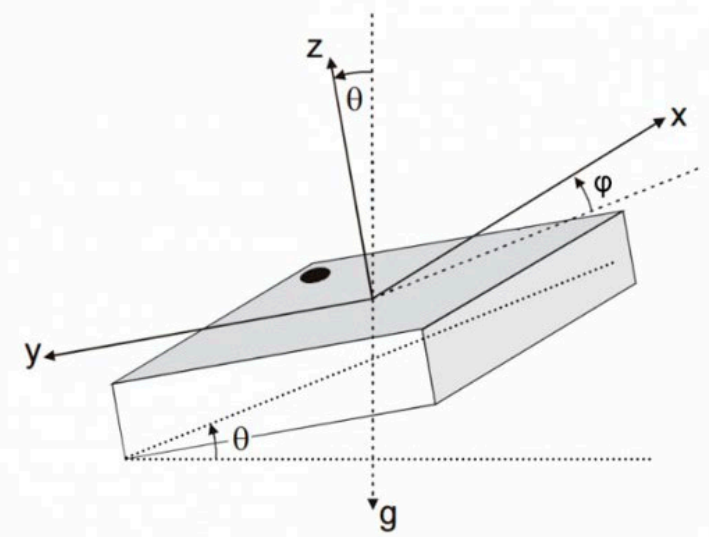

Fig. 3. Representación de las componentes de aceleración respecto del vector de aceleración de la gravedad (BMA220 Datasheet rev.1.10 Bosch Sensortec ${ }^{T M}$ )

sadores, uno para la captura de datos, y otro para la descarga de datos. En el diagrama se omite el conexionado de dicho panel.

\section{Técnicas Empleadas}

Como se mencionó anteriormente, tanto el acelerómetro como el giróscopo no acusan la posición del sistema por sí mismos. Para funcionar como sensores de posición necesitan un procesamiento de los datos de aceleración y velocidad que miden cada cierto tiempo. En este proyecto se utilizó como convención los ángulos de navegación (yaw, pitch, roll) para describir la orientación del sistema en tres dimensiones. La rotación alrededor del eje $\mathrm{z}$ (yaw) queda desafectada de la sensibilidad del acelerómetro, y solo puede ser detectada por el giróscopo, y por lo tanto las medidas de la misma con el MPU6050 no son muy precisas y se descartó su procesamiento. Además, en la marcha humana la mayor cantidad de movimiento está concentrada en el plano sagital (pitch) y en el plano frontal (roll).
En el caso del acelerómetro, los ángulos de inclinación se obtienen mediante fórmulas trigonométricas que transforman las mediciones de aceleración de los ejes x, y, z en los ángulos yaw, pitch, roll. Existen diversas fórmulas trigonométricas que cumplen este propósito. Para el presente trabajo se utilizó la formula publicada en la Nota de Aplicación de Freescale Semiconductor ${ }^{\mathrm{TM}}$ AN3461 Rev. 6, 03/2013:

$$
\begin{gathered}
\tan (\theta)=\frac{-a_{x}}{\sqrt{a_{y}^{2}+a_{z}^{2}}} \\
\tan (\varphi)=\frac{a_{y}}{a_{z}}
\end{gathered}
$$

Donde $\theta$ es el ángulo pitch, $\varphi$ el ángulo rolly $a_{x}, a_{y}, a_{z}$ la aceleración medida en cada eje.

A diferencia del acelerómetro, el giróscopo mide velocidad angular y por lo tanto para obtener el ángulo barrido correspondiente se debe realizar una integración. Distintos métodos de integración numérica son aplicables, como la Regla de los Trapecios o la Regla de Simpson. Es importante que la integración tenga un bajo costo de procesamiento, ya que permite mayores tasas de muestreo, lo que se traduce en una mayor exactitud. Por esta razón, se recurrió a la Regla del Rectángulo:

$$
\begin{aligned}
& \theta\left[n T_{s}\right]=\omega_{y} \cdot T_{s}+\theta\left[n T_{s}-1\right] \\
& \varphi\left[n T_{s}\right]=\omega_{x} \cdot T_{s}+\varphi\left[n T_{s}-1\right]
\end{aligned}
$$

Donde $\omega_{\mathrm{y}}$ es la velocidad angular medida en el eje $y, \omega_{x}$ la velocidad angular medida en el eje $\mathrm{x}, \mathrm{y} \mathrm{T}_{\mathrm{s}}$ el tiempo de muestreo. 


\section{Procesamiento de Datos}

Una vez establecidos los métodos que permiten al acelerómetro y al giróscopo acusar ángulos de posición, fue necesario programar la rutina de adquisición de datos en el microcontrolador.

Como se describió anteriormente, el dispositivo cuenta con tres módulos: microcontrolador (Atmega328p), memoria EEPROM (AT24C04C) y el módulo de sensores (MPU-6050). Los tres módulos están conectados al bus I2C, y la transferencia de datos es arbitrada por el microcontrolador, actuando de maestro, siendo la memoria y los sensores esclavos. Como las frecuencias a estudiar son relativamente bajas, la velocidad de transmisión de datos del bus no supone un discriminante, y se omitieron cálculos precisos para alcanzar la máxima velocidad, la cual además tiene asociada un mayor consumo.

Mediante programación y el protocolo $\mathrm{I} 2 \mathrm{C}$, el microcontrolador establece las configuraciones iniciales en los sensores, escribiendo en los registros del MPU-6050 reservados para ello. Se definió una sensibilidad de $\pm 8 \mathrm{~g}$ para el acelerómetro y de \pm 500 dps para el giróscopo.

Inicializados los sensores, se debió determinar correctamente la posición estática inicial, para que las inclinaciones que se midieran tuvieran certeza. Como esta posición de inclinación nula puede variar de un ensayo a otro, se debió calibrar el dispositivo cada vez que se realizó una prueba. La calibración tanto del acelerómetro como del giróscopo determina el offset de cada eje. El offset es calculado mediante el promedio de
5000 muestras del eje correspondiente. En esta etapa la tasa de muestreo es la máxima posible, siendo $\mathrm{T}_{\mathrm{s}}$ del orden de los microsegundos.

Determinado el offset en cada canal, se leen los registros del MPU-6050 que guardan las medidas del acelerómetro y el giróscopo, se le aplica la corrección de cero (offset) y finalmente se aplica la fórmula de transformación a los ángulos pitch y roll. Todo esto debe ocurrir en un lapso de tiempo constante y lo suficientemente corto para cumplir con el Teorema de Muestreo, y por lo tanto se estableció $\mathrm{T}_{\mathrm{s}}$ en $30 \mathrm{~ms}$.

La memoria EEPROM es de 1024 Bytes. Se complementó esta memoria con la memoria EEPROM interna del Atmega328p, cuya capacidad es de $1 \mathrm{~KB}$. Utilizando ambas memorias se tiene un espacio de $2 \mathrm{~KB}$ aproximadamente, y siendo que cada dato ocupa 2 bytes, se pueden capturar hasta 1000 muestras.

Los ángulos determinados por medio del giróscopo involucran una integración numérica, y por lo tanto tienen asociado un error de deriva (drift) inherente. Es por ello que se hace hincapié en que $\mathrm{T}_{\mathrm{s}}$ sea constante, para que la integración no introduzca error adicional.

La señal de cada canal del acelerómetro se filtró para minimizar el ruido. Se realizaron ensayos con un filtro FIR (promediador) y un IIR (primer orden), para evaluar el desempeño de cada uno.

El giróscopo, como se dijo anteriormente, posee alta inmunidad al ruido, por lo tanto, no precisa de un filtrado. 


\section{Ensayos}

Se eligió como plataforma de pruebas un péndulo mecánico, colocando el dispositivo sensor solidario al brazo rígido de dicho péndulo. Esto permitió realizar pruebas tanto dinámicas como estáticas, y así determinar completamente la precisión, la exactitud, la linealidad y los errores del instrumento.

Se decidió medir el ángulo roll, ya que los resultados se extendían para el ángulo pitch, e incluso para cualquier tipo de ángulo.

El procedimiento de los ensayos dinámicos consistió en iniciar el registro de datos en la posición de inclinación nula, trasladar el péndulo a la posición de $90^{\circ}$, y soltarlo para que el efecto de la gravedad describa sobre el instrumento un movimiento oscilatorio amortiguado.

Las mediciones dinámicas realizadas con el acelerómetro siguiendo dicho procedimiento fueron:

1) Medición del valor crudo del vector de aceleración de la gravedad en el eje z.

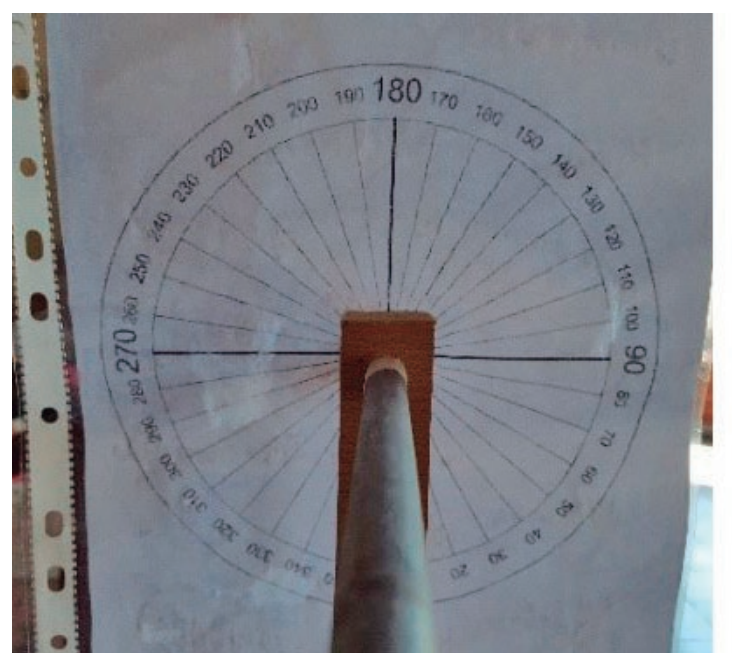

2) Medición del ángulo roll, sin filtrar.

3) Medición del ángulo roll, con filtro FIR del tipo promediador con 10 coeficientes.

4) Medición del ángulo roll, con filtro IIR de primer orden.

En el caso del giróscopo, las pruebas realizadas con dicho procedimiento fueron:

1) Medición del ángulo roll.

El procedimiento de los ensayos estáticos consistió en iniciar la captura de datos también en la posición de inclinación nula, y luego trasladar a una velocidad constante el péndulo a distintas posiciones elegidas por el operador, marcando generalmente dos o tres ángulos.

Las mediciones estáticas realizadas con el acelerómetro siguiendo dicho procedimiento fueron:

1) Medición del ángulo roll, con filtro FIR del tipo promediador con 10 coeficientes.

2) Medición del ángulo roll, con filtro IIR de primer orden.

En el caso del giróscopo, las pruebas realizadas con dicho procedimiento fueron:

1) Medición del ángulo roll.

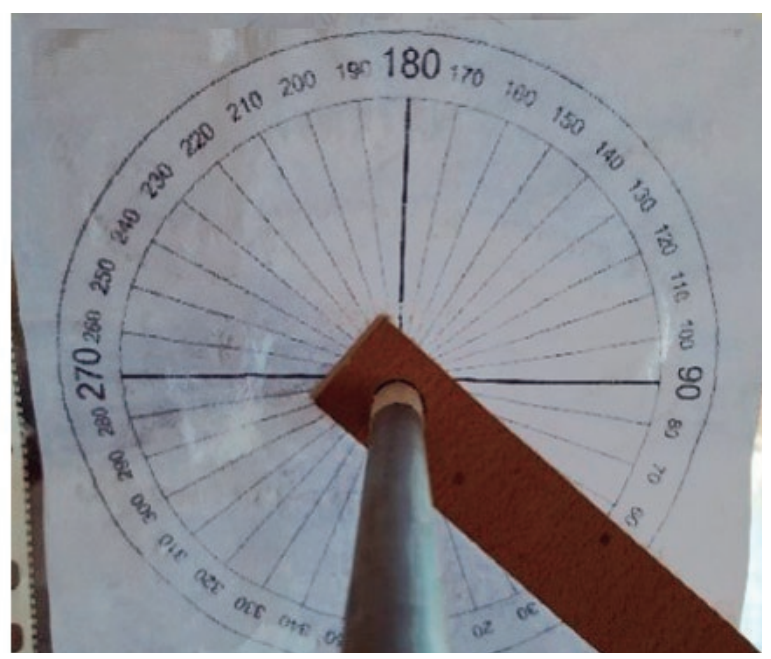

Fig. 4. Imagen que ilustra el péndulo mecánico utilizado 
Además, se realizaron dos pruebas adicionales, una dinámica y otra estática, combinando ambos, acelerómetro y giróscopo, mediante un filtro complementario.

Las mediciones obtenidas se contrastaron con una circunferencia graduada cuyo centro coincidía con el eje de rotación del péndulo, y también con una aplicación Android de uso libre para teléfono móvil, que permitía utilizar a dicho teléfono como nivel de inclinación.

La aplicación Android hace uso de los acelerómetros incorporados en el Smartphone para determinar la inclinación del mismo. El móvil con la aplicación funcionando se colocó solidario al brazo del péndulo, de manera que describa el mismo movimiento que el dispositivo y sirva como patrón para validar las mediciones.

\section{RESULTADOS}

A continuación, se exponen las gráficas que arrojaron los ensayos detallados en el apartado anterior. En algunas pruebas se tomaron 500 muestras, y en otras 1000 muestras, según la información necesaria para una correcta visualización del fenómeno.

\section{Pruebas Dinámicas del Acelerómetro}
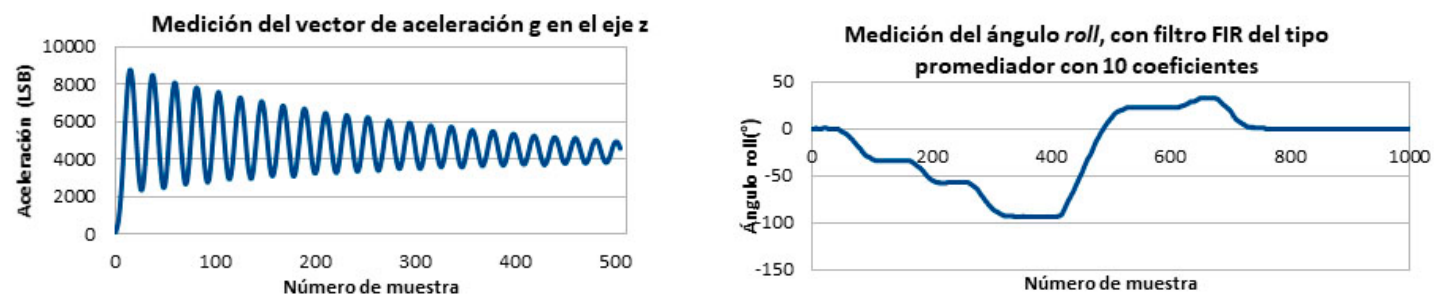

4. Pruebas Estáticas del Giróscopo

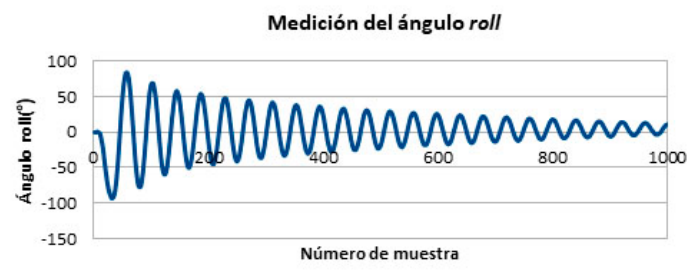

3. Pruebas Estáticas del Acelerómetro
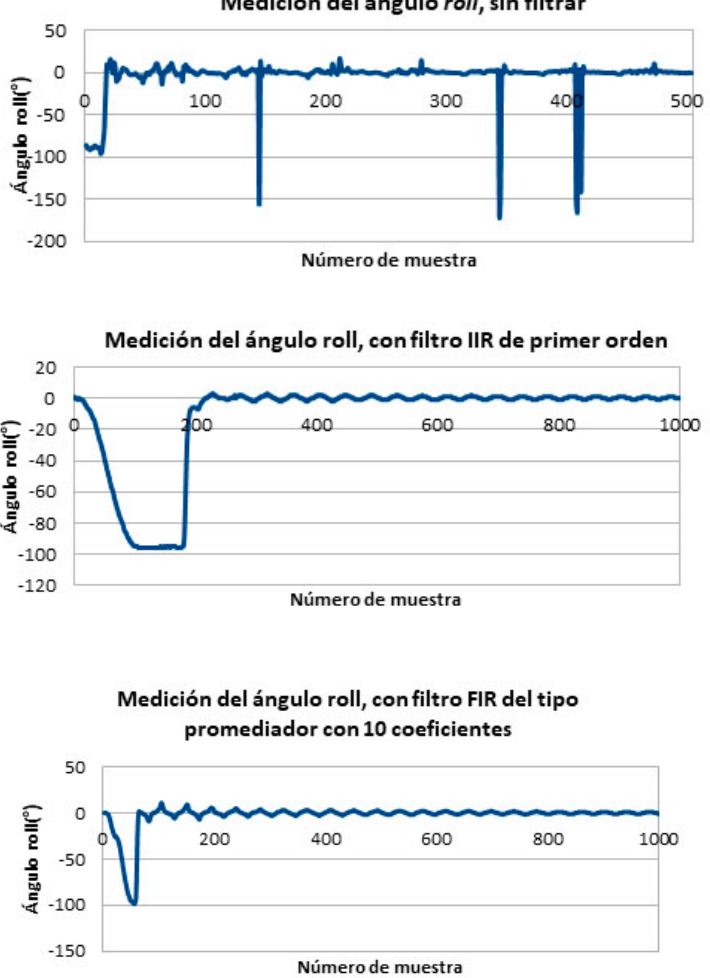

2. Pruebas Dinámicas del Giróscopo 


\section{Pruebas Estáticas del Giróscopo}

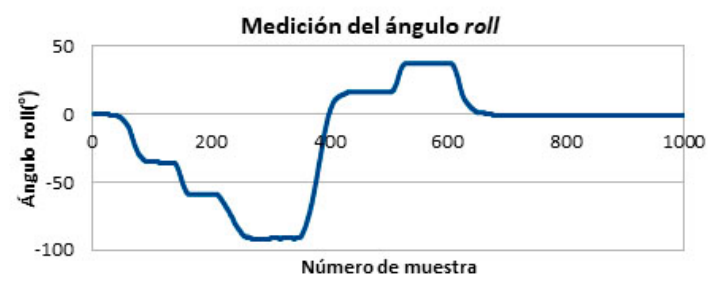

\section{Discusión}

1. Pruebas Dinámicas del Acelerómetro

1) Medición del valor crudo del vector de aceleración de la gravedad en el eje z:

Este ensayo se realizó para comprobar la respuesta del acelerómetro a la gravedad. Los datos obtenidos describieron el movimiento oscilatorio amortiguado, con un valor medio de 4096 LSB aproximadamente, el cual es el valor crudo de aceleración correspondiente a $1 \mathrm{~g}$, para la sensibilidad seleccionada.

2) Medición del ángulo roll, sin filtrar:

El objetivo era observar el movimiento oscilatorio amortiguado, sin tratar la señal y observar el efecto del ruido en las muestras. Sólo se detectó en ángulo de inicio de $-90^{\circ}$, y luego el acelerómetro no pudo seguir el movimiento. En esta prueba queda demostrado la necesidad de un filtrado, ya que el nivel de perturbaciones podía contribuir a una información errónea.

3) Medición del ángulo roll, con filtro FIR del tipo promediador con 10 coeficientes:

Con la aplicación de un filtro promediador de 10 coeficientes, las perturbaciones son prácticamente eliminadas. El acelerómetro sigue correctamente el movimiento durante la traslación paulatina desde $0^{\circ}$ a $-90^{\circ}$, pero pierde respuesta al comienzo del movimiento oscilatorio amortiguado aplicado por la gravedad.

4) Medición del ángulo roll, con filtro IIR de primer orden:

Aquí se comprobó que un filtro IIR de primer orden cuyo costo de programación es menor, cumple correctamente con los requisitos de una señal libre de ruido. En este caso la posición de $-90^{\circ}$ se mantuvo estacionaria un cierto tiempo para contrastarlo con el patrón. Se obtuvo un error de $<1^{\circ}$. Luego de tres ensayos tratando de reproducir la dinámica del movimiento del péndulo, se comprueba la hipótesis de que el acelerómetro no es apto para seguir movimientos rápidos.

\section{Pruebas Dinámicas del Giróscopo}

1) Medición del ángulo roll:

Como se esperaba, el giróscopo reprodujo correctamente la dinámica de movimiento involucrada en el ensayo. Sin necesidad de filtrado presento una señal sin perturbaciones, destacando su inmunidad al ruido. Como también se predijo, a la larga existe un error de deriva (drift). El mismo se manifiesta como un valor medio distinto de $0^{\circ}$ en las últimas muestras.

\section{Pruebas Estáticas del Acelerómetro}

1) Medición del ángulo roll, con filtro FIR del tipo promediador con 10 coeficientes:

Siguiendo el procedimiento, se marcaron distintos ángulos con el péndulo, y se contrastaron con la circunferencia graduada y el medidor de inclinación patrón del teléfono móvil. El error observado fue de $<1^{\circ}$ en todas las mediciones. 
2) Medición del ángulo roll, con filtro IIR de primer orden:

Las mediciones no sufren cambios al utilizar el filtro IIR. El error observado también fue de $<1^{\circ}$.

\section{Pruebas Estáticas del Giróscopo}

1) Medición del ángulo roll:

El giróscopo se desenvolvió correctamente en las mediciones estáticas. El error de cada medida fue de $<1^{\circ}$, y el error de deriva (drift) fue de $-1^{\circ}$, significativamente menor que en el caso dinámico, debido a que se pasó pocas veces por el punto de $0^{\circ}$.

Para compensar la debilidad de un sensor con la fortaleza de otro, es posible recurrir a un filtro complementario. Se realizó una prueba dinámica y una estática aplicando dicho filtro y combinando las salidas del acelerómetro y el giróscopo.

5. Prueba Dinámica y Estática con Filtro Complementario
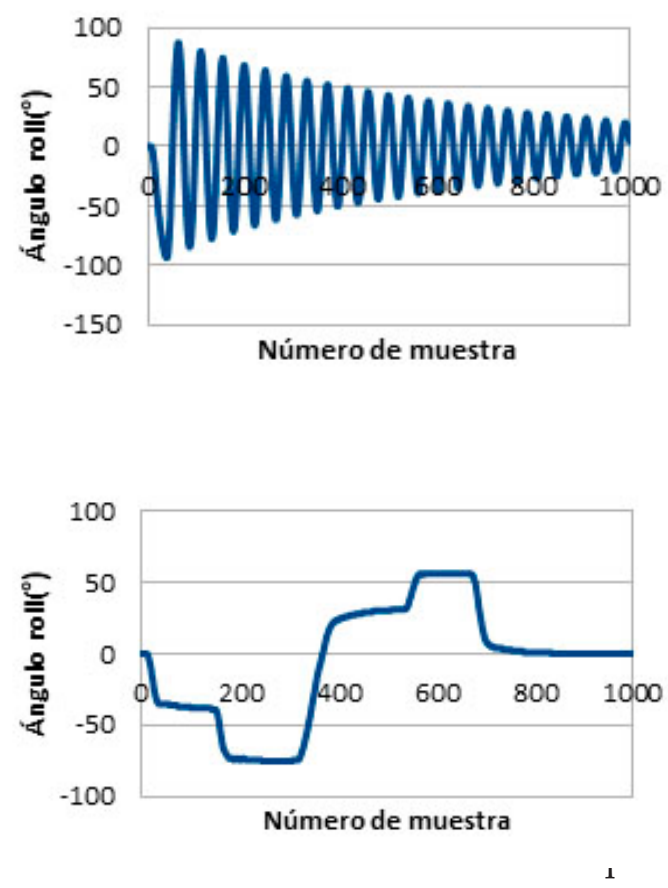

acelerómetro en tiempos largos corrige el error de deriva del giróscopo, resultando una medición más robusta. En el caso estático se mantiene el error de $<1^{\circ}$ y la señal no presenta deriva.

\section{CONCLUSIÓN}

Mediante el estudio previo de los dispositivos involucrados y el posterior análisis detallado de los resultados arrojados por los ensayos programados, se logró la correcta caracterización tanto del acelerómetro como el giróscopo. Presentan característica lineal, repetitividad en la medida y pequeño error.

Teniendo esto en cuenta, se comprueba la hipótesis postulada en el inicio de este trabajo. Ambos sensores pueden ser utilizados correctamente como indicadores de posición para medir la inclinación de miembros en estudios de biomecánica.

El mayor potencial del acelerómetro y el giróscopo sale a la luz cuando se combinan mediante un filtro complementario, presentando una medida ausente de deriva $\mathrm{y}$ de errores en tiempos cortos.

$\mathrm{El}$ instrumento de medición al ser inalámbrico, mediante las correspondientes actualizaciones apunta tanto al uso tipo Holter como al uso en laboratorio de marcha. Es interesante la ventaja de un uso cotidiano, ya que elimina el error introducido por el paciente de tender innato a corregir su marcha cuando se siente observado. Un modelo de este tipo es viable reemplazando la memoria EEPROM por una SD de mayor capacidad, y aplicando un cálculo de consumo estricto para mayor eficiencia de la batería. 


\section{BIBLIOGRAFÍA}

1. MPU-6000 and MPU-6050 Product Specification Revision 3.4, Document Number: PSMPU-6000A-00, 19/08/2013, InvenSense ${ }^{\mathrm{TM}}$.

2. MPU-6000 and MPU-6050 Register Map and Descriptions Revision 4.2, Document Number: RM-MPU-6000A-00, 19/08/2013, InvenSen$\mathrm{se}^{\mathrm{TM}}$.

3. 8-bit AVR Microcontroller with 4/8/16/32K Bytes In-System Programmable Flash ATmega48P/88P/168P/328P Datasheet Revi- sion 8025FS, 08/2008, Atmel $^{\mathrm{TM}}$.

4. SEEPROM AT24C04C/08C Datasheet Revision 8787F, 12/2016, Atmel ${ }^{\mathrm{TM}}$.

5. BMA220 Datasheet Revision 1.10, Document Number: BST-BMA220-DS003-07, 13/04/2011, Bosch Sensortec ${ }^{\mathrm{TM}}$.

6. "Tilt Sensing Using a Three-Axis Accelerometer" Revision 6, Document Number: AN3461, 03/2013, Application Note Freescale Semiconductors $^{\mathrm{TM}}$. 